ISSN 1813-548X

\title{
Technique de datation des échantillons riches en uranium par les séries de l'uranium en utilisant la spectrométrie gamma à basse énergie
}

\section{A. Choukri”, O.-K. Hakam et M. Zairi}

Laboratoire de physique de la matière et rayonnement, UFR faibles radioactivités, physique mathématique et environnement, Equipe de physique et techniques nucléaires, Université Ibn Tofail, Faculté des sciences, B.P. 133, 14 000, Kénitra, Maroc.

(Reçu le 02 Mai 2006, accepté le 15 Novembre 2006)

*Correspondance, courriel : choukrimajid@yahoo.com

\section{Résumé}

Dans ce travail, une technique de datation par les séries de l'uranium utilisant la spectrométrie gamma à basse énergie $(<100 \mathrm{keV})$ a été améliorée de façon qu'elle soit applicable aux échantillons riches en uranium. Elle consiste à réduire l'échantillon en un précipité d'hydroxydes renfermant les isotopes de l'uranium et du thorium. Cette technique a permis de mesurer directement dans un échantillon géologique riche en uranium, et sans aucune correction due à l'autoabsorption ou à la géométrie de comptage et aux interférences des raies gamma, les rapports ${ }^{230} \mathrm{Th} /{ }^{234} \mathrm{U},{ }^{234} \mathrm{U} /{ }^{238} \mathrm{U}$ et ${ }^{231} \mathrm{~Pa} /{ }^{235} \mathrm{U}$ nécessaires pour dater un échantillon par les méthodes de déséquilibre radioactif dans les familles de l'uranium ${ }^{230} \mathrm{Th} /{ }^{234} \mathrm{U}$, et ${ }^{231} \mathrm{~Pa} /{ }^{235} \mathrm{U}$. Les rapports et l'âge mesurés par cette technique sont comparables à ceux obtenus à l'aide de la spectrométrie alpha convenable pour ce genre de mesures.

La spectrométrie gamma à basse énergie destructive a permis, dans ce travail, de dater un échantillon riche en uranium, à partir du même 
spectre, par 2 méthodes de déséquilibre radioactif ${ }^{230} \mathrm{Th} /{ }^{234} \mathrm{U}$ et ${ }^{231} \mathrm{~Pa} /{ }^{235} \mathrm{U}$ ); ce qui permettra d'éviter le problème de contamination radioactive en spectrométrie alpha dans les laboratoires qui traitent aussi les échantillons de faibles activités.

Mots-clés : Datation, spectrométrie gamma, uranium, urano-vanadate, pechblende.

\section{Abstract \\ Dating technique of samples rich in uranium by the series of the uranium by using the gamma spectrometry with low energy}

In this work, a dating technique by the series of the uranium using the gamma spectrometry with low energy $(<100 \mathrm{keV})$ was improved so that it could be applicable to samples rich in uranium. It consists in reducing the sample to a hasty of hydroxides containing the isotopes of the uranium and the thorium. This technique allowed to measure directly in a geologic sample rich in uranium, and without any correction due to the autoabsorption or to the geometry of counting and to the interferences of the lines gamma, ${ }^{230} \mathrm{Th} /{ }^{234} \mathrm{U},{ }^{234} \mathrm{U} /{ }^{238} \mathrm{U}$ and ${ }^{231} \mathrm{~Pa} /{ }^{235} \mathrm{U}$ activity ratios necessary to date a sample by the methods of radioactive imbalance in the families of the uranium ${ }^{230} \mathrm{Th} /{ }^{234} \mathrm{U}$, and ${ }^{231} \mathrm{~Pa} /{ }^{235} \mathrm{U}$ ). Activity ratios and age measured by this technique are comparable to those obtained by means of the suitable alpha spectrometry for this kind of measures.

The gamma spectrometry with low destructive energy allowed, in this work, to date a sample rich in uranium, from the same spectrum, by 2 methods of radioactive imbalance $\left({ }^{230} \mathrm{Th} /{ }^{234} \mathrm{U}\right.$ and $\left.{ }^{231} \mathrm{~Pa} /{ }^{235} \mathrm{U}\right)$. What will allow to avoid the radioactive pollution problem for laboratories which also handle the samples of weak activities

Keywords : Dating, gamma spectrometry, uranium, urano-vanadate, petchblende. 


\section{Introduction}

En archéologie, les os et dents des restes humains et animaux ont été datés par les méthodes de datation basées sur les déséquilibres radioactifs dans les familles de l'uranium ${ }^{230} \mathrm{Th} /{ }^{234} \mathrm{U}$ et $\left.{ }^{231} \mathrm{~Pa} /{ }^{235} \mathrm{U}\right)$ en utilisant, soit la spectrométrie alpha, soit la spectrométrie gamma.

Contrairement aux rayons alpha, les rayons gamma ont un long parcours dans la matière. La spectrométrie gamma ne nécessite donc pas de préparation d'échantillons minces. Après le progrès des détecteurs $\mathrm{Ge}-\mathrm{Li}$ et Ge haute pureté pour les hautes et les faibles énergies, des séries de mesures et de datations ont été effectuées par spectrométrie gamma non destructive [1-5].

Ces auteurs ont utilisé cette technique, ne nécessitant aucun traitement de l'échantillon surtout pour dater les ossements fossilisés provenant des sites préhistoriques. Ils l'ont étendue après à la datation d'autres échantillons tels que les sédiments marins, les nodules de manganèse et les coraux. La spectrométrie gamma non destructive présente l'avantage de ne pas détruire l'échantillon; par contre elle nécessite des corrections dues à l'autoabsorption dans l'échantillon, la géométrie de comptage et l'interférence des pics dans le spectre gamma. La raie $53,2 \mathrm{keV}$ utilisée pour mesurer II ${ }^{234} \mathrm{U}$ est due aussi au ${ }^{214} \mathrm{~Pb}$. Le calcul de l'activité de I' ${ }^{234} \mathrm{U}$, s'obtient par soustraction de la contribution $d \mathrm{du}^{214} \mathrm{~Pb}$. Celle-ci étant importante (l'intensité de cette raie est 10 fois plus grande pour ${ }^{214} \mathrm{~Pb}$ que pour ${ }^{234} \mathrm{U}$ ). La précision de la mesure est très médiocre.

Les échantillons géologiques riches en uranium constituent un matériel idéal pour l'analyse par spectrométrie gamma non destructive. Par contre, ils présentent, en spectrométrie alpha, l'inconvénient de pollution et de contamination radioactive du matériel utilisé dans les laboratoires qui traitent les échantillons de faibles radioactivités telles que les formations marines et continentales.

On propose, dans ce travail, une nouvelle technique destructive pour analyser et dater les échantillons géologiques riches en uranium par spectrométrie gamma à basse énergie. Elle consiste à réduire l'échantillon à un précipité d'hydroxydes renfermant les isotopes de

\section{A. Choukri et al.}


l'uranium et du thorium. Cette technique permettra de mesurer directement et sans aucune correction due à l'autoabsorption ou à la géométrie de comptage et aux interférences des raies gamma, les rapports ${ }^{230} \mathrm{Th} /{ }^{234} \mathrm{U},{ }^{234} \mathrm{U} /{ }^{238} \mathrm{U}$ et ${ }^{231} \mathrm{~Pa} /{ }^{235} \mathrm{U}$ nécessaires pour dater un échantillon par les méthodes de déséquilibre radioactif dans les familles de l'uranium ${ }^{230} \mathrm{Th} /{ }^{234} \mathrm{U}$, et ${ }^{231} \mathrm{~Pa} /{ }^{235} \mathrm{U}$.

\section{Partie expérimentale}

\section{2-1. choix du détecteur et préparation des échantillons}

Les raies gamma utilisées dans la mesure des rapports ${ }^{230} \mathrm{Th} /{ }^{234} \mathrm{U}$, ${ }^{234} \mathrm{U}{ }^{238} \mathrm{U}$ et ${ }^{231} \mathrm{~Pa}{ }^{235} \mathrm{U}$ sont représentées dans le Tableau 1. Elles sont toutes de très faible énergie et, en plus, leurs énergies sont très proches les unes des autres. Elles sont situées entre 25,6 keV et $67,7 \mathrm{keV}$, c'est pourquoi il a été nécessaire de choisir un détecteur qui puisse satisfaire simultanément deux impératifs fondamentaux : un très grand pouvoir de résolution et une très grande efficacité. Pour cela, nous avons utilisé un détecteur performant germanium haute pureté type $P$ qui convient parfaitement à la détection des rayons $X$ et des gamma à faible énergie. Il est fabriqué par une technique d'implantation ionique, sa couche morte extrêmement mince et sa relativement grande surface permettent de détecter des rayonnements gamma de très faible énergie avec une grande sensibilité et une excellente résolution.

La spectrométrie gamma non destructive consiste à la préparation des échantillons étalons en des dimensions, des poids et des concentrations en uranium similaires à ceux des échantillons à analyser. La nouvelle technique qu'on propose consiste à dissoudre totalement les deux échantillons dans une solution acide, ajouter la même quantité de fer dans les deux solutions avant de filtrer l'ensemble et précipiter les hydroxydes de fer à l'aide de l'ammoniaque à pH 7 à 8 . L'uranium et le thorium sont quantitativement précipités avec le fer, tandis que le radium et ses descendants, principaux émetteurs gamma dans un spectre

\section{A. Choukri et al.}


uranifère, restent en solution. Le radium peut être récupéré par précipitation avec les carbonates de baryum. Le précipité contenant l'uranium et le thorium est ensuite mis dans une petite boite en plastique ayant les mêmes dimensions que la fenêtre du détecteur et dont l'épaisseur de la paroi est très mince pour minimiser l'absorption des rayonnements à faible énergie par le plastique.

Tableau 1 : Raies utilisées dans le calcul des rapports d'équilibre ${ }^{230} \mathrm{Th}^{2{ }^{34}} \mathrm{U},{ }^{234} \mathrm{U}{ }^{238} \mathrm{U}$ et ${ }^{231} \mathrm{~Pa}{ }^{235} \mathrm{U}$.

\begin{tabular}{|c|c|c|c|}
\hline Raie $(\mathrm{keV})$ & Élément émetteur & Élément mesuré & Intensité $(\%)$ \\
\hline 25,6 & ${ }^{231} \mathrm{Th}$ & ${ }^{235} \mathrm{U}$ & 14,5 \\
\hline 27,4 & ${ }^{231} \mathrm{~Pa}$ & ${ }^{231} \mathrm{~Pa}$ & 9,52 \\
\hline 50,2 & ${ }^{227} \mathrm{Th}$ & ${ }^{231} \mathrm{~Pa}$ & 8,50 \\
\hline 53,2 & ${ }^{234} \mathrm{U}$ & ${ }^{234} \mathrm{U}$ & 0,119 \\
\hline 53,3 & ${ }^{214} \mathrm{~Pb}$ & & 1,10 \\
\hline 63,3 & ${ }^{234} \mathrm{Th}$ & ${ }^{238} \mathrm{U}$ & 3,81 \\
\hline 67,7 & ${ }^{230} \mathrm{Th}$ & ${ }^{230} \mathrm{Th}$ & 0,378 \\
\hline
\end{tabular}

\section{2-2. Echantillonnage}

Dans le but de tester la technique proposée, on a utilisé 2 échantillons géologiques riches en uranium, le premier est un échantillon étalon de pechblende ancienne dont tous les radioéléments sont déjà en équilibre, le deuxième échantillon est un dépôt superficiel jaune formé de cristaux fins sur une roche prélevée du plateau phosphatier de Ganntour au Maroc. L'examen de ce dépôt par diffraction $\mathrm{X}$ a montré qu'il s'agissait d'un mélange d'urano-vanadate de calcium appelé la Tyuyamunite de formule $\mathrm{Ca}\left(\mathrm{UO}_{2}\right)_{2}\left(\mathrm{VO}_{4}\right) 2,8 \mathrm{H}_{2} \mathrm{O}$ et d'un urano-vanadate d'aluminium qui serait la vanuralite de formule $\mathrm{Al}\left(\mathrm{UO}_{2}\right)_{2}\left(\mathrm{VO}_{4}\right)_{2} \mathrm{OH} .11 \mathrm{H}_{2} \mathrm{O}$.

Les teneurs en uranium de ces deux échantillons ont été déterminées par une technique non destructive utilisant un détecteur germanium pur planaire à haute résolution pour les hautes énergies. Les concentrations de l'uranium ont été évaluées à $0,28 \pm 0,02 \%$ pour l'échantillon de pechblende et à $6,53 \pm 0,21 \%$ pour l'échantillon d'urano-vanadate.

\section{A. Choukri et al.}




\section{Résultats et discussion}

\section{3-1. Mesure des rapports d'équilibre radioactif}

Les rapports radioactifs dans l'échantillon d'urano-vanadate ont été mesurés par trois techniques différentes: la spectrométrie gamma à basse énergie non destructive, la spectrométrie gamma à basse énergie destructive et par spectrométrie alpha.

\section{3-1-1. La spectrométrie gamma à basse énergie non destructive}

Parmi les rapports utilisés pour la datation par les séries de l'uranium, seuls les rapports ${ }^{230} \mathrm{Th} /{ }^{238} \mathrm{U}$ et ${ }^{231} \mathrm{~Pa} /{ }^{235} \mathrm{U}$ ont été mesurés avec précision. Les facteurs d'autoabsorption ont été déterminés en étudiant l'absorption dans les matrices des deux échantillons, des raies d'intérêt émises par des sources étalons.

Les rapports ${ }^{230} \mathrm{Th} /{ }^{238} \mathrm{U}$ et ${ }^{231} \mathrm{~Pa} /{ }^{235} \mathrm{U}$ mesurés directement dans l'échantillon d'urano-vanadate sont représentés dans le Tableau 2. Ils ont été corrigés par normalisation aux mêmes rapports dans l'échantillon étalon de pechblende. Les deux rapports sont mesurés chacun à partir des raies d'énergie voisines dans la région à basse énergie, minimisant ainsi les erreurs de mesure dues à l'évaluation des facteurs d'autoabsorption.

Tableau 2 : Résultats de la spectrométrie gamma à basse énergie non destructive

\begin{tabular}{|c|c|c|}
\hline Rapports d'équilibre & Rapports des raies & Résultats \\
\hline${ }^{231} \mathrm{~Pa} /{ }^{235} \mathrm{U}$ & $27,4(\mathrm{keV}) / 25,6(\mathrm{keV})$ & $0,62 \pm 0,06$ \\
\hline${ }^{230} \mathrm{Th} /{ }^{238} \mathrm{U}$ & $67,7(\mathrm{keV}) / 63,3(\mathrm{keV})$ & $0,33 \pm 0,03$ \\
\hline
\end{tabular}

3-1-2. La spectrométrie gamma à basse énergie destructive

La mesure de l'activité de $I^{1234} U$, dans un échantillon, par soustraction de la contribution $d u^{214} \mathrm{~Pb}$ sous le pic d'énergie $53,2 \mathrm{keV}$, ne permet pas un

A. Choukri et al. 
calcul précis du rapport ${ }^{234} \mathrm{U} /{ }^{238} \mathrm{U}$. La mesure directe de $\mathrm{I}^{1234} \mathrm{U}$ par la raie 53,2 keV nécessite donc une élimination du radium et de ses descendants de la matrice de l'échantillon à dater et de l'échantillon étalon afin d'éviter les corrections dues à l'interférence de $I^{1234} \mathrm{U}$ avec le ${ }^{214} \mathrm{~Pb}$ sous l'unique raie émise par ${ }^{1234} \mathrm{U}$.

Après dissolution des deux échantillons par $\mathrm{HNO}_{3}$ concentré et précipitation des hydroxydes de fer, on a obtenu des spectres gamma dont les pics dans la région à basse énergie, sont bien résolus et sortent nettement du bruit de fond grâce à l'absence des descendants $\mathrm{du}{ }^{226} \mathrm{Ra}$ et à la réduction de masse de l'échantillon sous forme d'hydroxydes de fer. Les autres éléments majeurs ( $\mathrm{Al}, \mathrm{Ca}, \mathrm{Mg}$, etc... ) ainsi que le radium passent en solution et les descendants du radium à courte période décroissent rapidement [3,6-10]. L'absence du radium et de ses descendants fait baisser considérablement le bruit de fond dans la région à basse énergie; ce qui permet l'amélioration de la précision de mesure des rapports d'équilibre et des âges.

La précision de mesure de chaque raie dépend de son intensité d'émission, de l'activité de l'échantillon et de l'état d'équilibre entre le radioélément émetteur et son chef de filiation. Elle dépend aussi de la performance du détecteur et des conditions de comptage. Pour des échantillons moins actifs, on peut soit augmenter le poids de l'échantillon, soit compter pendant plus de temps.

Le Tableau 3 présente les rapports d'activité mesurés dans l'échantillon d'urano-vanadate par comparaison avec l'échantillon de pechblende à l'équilibre. Notons que l'activité ${ }^{231} \mathrm{~Pa} /{ }^{235} \mathrm{U}$ en spectrométrie gamma destructive a été obtenue à partir de ${ }^{227} \mathrm{Th} /{ }^{235} \mathrm{U}$ parce qu'il a été remarqué que ${ }^{231} \mathrm{~Pa}$ ne précipitait pas toujours totalement avec les hydroxydes de fer comme l'uranium et le thorium.

Les rapports ${ }^{230} \mathrm{Th} /{ }^{238} \mathrm{U}$ et ${ }^{231} \mathrm{~Pa} /{ }^{235} \mathrm{U}$ sont obtenus avec des précisions meilleures que les rapports ${ }^{234} \mathrm{U} /{ }^{238} \mathrm{U}$ et ${ }^{230} \mathrm{Th} /{ }^{234} \mathrm{U}$ à cause de la faible intensité de la raie $53,2 \mathrm{keV}(0,119 \%)$ utilisée pour mesurer $1^{1234} \mathrm{U}$; ce qui fera que la datation par spectrométrie gamma à basse énergie 
destructive est plus précise par la méthode ${ }^{231} \mathrm{~Pa} /{ }^{235} \mathrm{U}$ que par la méthode ${ }^{230} \mathrm{Th} /{ }^{234} \mathrm{U}$.

Tableau 3 : Rapports d'activités moyens corrigés dans l'échantillon d'urano-vanadate et précision de mesure

\begin{tabular}{c|c|c} 
& & Précision (\%) \\
\hline${ }^{230} \mathrm{Th} /{ }^{238} \mathrm{U}$ & $0,286 \pm 0,010$ & 3,46 \\
\hline${ }^{234} \mathrm{U} /{ }^{238} \mathrm{U}$ & $0,791 \pm 0,055$ & 6,97 \\
\hline${ }^{230} \mathrm{Th} /{ }^{234} \mathrm{U}$ & $0,362 \pm 0,027$ & 7,56 \\
\hline${ }^{231} \mathrm{~Pa} /{ }^{235} \mathrm{U}$ & $0,540 \pm 0,021$ & 3,94
\end{tabular}

3-1-3. Comparaison des résultats de la spectrométrie gamma avec les résultats de la spectrométrie alpha

L'échantillon d'urano-vanadate a été analysé par spectrométrie alpha et seulement quelques milligrammes de cet échantillon riche en uranium ont été suffisants. L'uranium et le thorium ont été séparés et purifiés selon la procédure décrite par Choukri [11] et Berrada et al. [12]. L'activité des dépôts de l'uranium et du thorium a été mesurée à l'aide d'un détecteur à circulation de gaz, de type chambre à grille, ce qui a permis de mesurer avec précision les activités alpha des isotopes de l'uranium et de thorium. Le ${ }^{231} \mathrm{~Pa}$ a été mesuré par l'intermédiaire $d u^{227} T h$ supposé en équilibre avec lui. Les résultats d'analyse de l'échantillon d'urano-vanadate par spectrométrie alpha sont donnés dans le Tableau 4.

Tableau 4 : Résultats de la spectrométrie alpha pour l'échantillon d'urano-vanadate

\begin{tabular}{c|c|c} 
& & Précision (\%) \\
\hline${ }^{238} \mathrm{U}(\mathrm{ppm})$ & $7,2 \pm 0,3$ & 4,2 \\
\hline${ }^{230} \mathrm{Th} /{ }^{238} \mathrm{U}$ & $0,292 \pm 0,009$ & 3,1 \\
\hline${ }^{230} \mathrm{Th} /{ }^{234} \mathrm{U}$ & $0,365 \pm 0,011$ & 3,0 \\
\hline${ }^{234} \mathrm{U} /{ }^{238} \mathrm{U}$ & $0,801 \pm 0,007$ & 0,9 \\
\hline${ }^{231} \mathrm{~Pa} /{ }^{235} \mathrm{U}$ & $0,543 \pm 0,024$ & 4,4
\end{tabular}

A. Choukri et al. 
Les incertitudes des données sont des déviations standard de 5 mesures indépendantes pour le rapport ${ }^{234} \mathrm{U}{ }^{238} \mathrm{U}$ et de 3 mesures pour les autres rapports tenant compte des incertitudes sur les comptages et des incertitudes provenant des différentes manipulations chimiques.

Une comparaison des résultats obtenus à l'aide des deux techniques montre que la spectrométrie gamma à basse énergie destructive et la spectrométrie alpha donnent pratiquement les mêmes rapports d'équilibre dans l'échantillon d'urano-vanadate avec des précisions du même ordre de grandeur pour les rapports ${ }^{230} \mathrm{Th} /{ }^{238} \mathrm{U}$ et ${ }^{231} \mathrm{~Pa} /{ }^{235} \mathrm{U}$, par contre les rapports ${ }^{230} \mathrm{Th} /{ }^{234} \mathrm{U}$ et ${ }^{234} \mathrm{U} /{ }^{238} \mathrm{U}$ donnés par la spectrométrie gamma à basse énergie destructive sont moins précis qu'en spectrométrie alpha à cause de la mesure de ${ }^{234} \mathrm{U}$ par la raie $53,2 \mathrm{keV}$ de faible intensité.

\section{3-2. Datation et interprétation de l'âge de l'échantillon d'urano- vanadate}

Les âges par les méthodes ${ }^{230} \mathrm{Th} /{ }^{234} \mathrm{U}$ et ${ }^{231} \mathrm{~Pa} /{ }^{235} \mathrm{U}$ de la formation de l'échantillon d'urano vanadate fournis par les trois techniques utilisées sont donnés dans le Tableau 4.

La spectrométrie gamma à basse énergie non destructive ne permet de dater que par une seule méthode $\left.{ }^{231} \mathrm{~Pa} /{ }^{235} \mathrm{U}\right)$ or, en absence de données géologiques, géomorphologiques et paléoclimatologiques capables dans certains cas de situer l'échantillon sur une échelle de temps stratigraphique, une deuxième méthode est indispensable pour juger la fermeture du système et valider l'âge obtenu.

Tableau 4 : Ages ${ }^{230}$ Th et ${ }^{231} \mathrm{~Pa}$ de l'échantillon d'urano-vanadate

\begin{tabular}{|l|l|l|l|}
\hline & \multicolumn{1}{c|}{$\boldsymbol{\gamma}$ non dest. } & \multicolumn{1}{c|}{$\boldsymbol{\gamma}$ dest. } & \multicolumn{1}{c|}{$\boldsymbol{\alpha}$} \\
\hline Age 230Th/234 & & $49,9+5,5$ & $50,3+2,1$ \\
\hline (ka) & & $-5,0$ & $-2,0$ \\
\hline Age 231Pa/235U & $45,4 \pm 7,4$ & $38,5+2,3$ & $38,8+2,7$ \\
\hline (ka) & & $-2,2$ & $-2,5$ \\
\hline
\end{tabular}


Le Tableau 4 montre que l'on obtient pratiquement les mêmes résultats par la spectrométrie gamma à basse énergie destructive et par la spectrométrie alpha. Les deux techniques confirment que les âges ${ }^{230} \mathrm{Th}$ et ${ }^{231} \mathrm{~Pa}$ sont en désaccord, ce qui signifie que le cristal de l'uranovanadate était un système ouvert. Plusieurs scénarios d'échange avec le milieu environnant peuvent cependant être envisagés. Un apport ultérieur d'uranium sans ses descendants ne peut que faire rajeunir l'âge du cristal, par contre un départ le fait vieillir.

\section{Conclusion}

La technique destructive proposée pour mesurer les rapports d'équilibre dans les échantillons géologiques a permis de résoudre 2 problèmes majeurs souvent attribués à la spectrométrie gamma non destructive :

- Le radium est éliminé en solution, le ${ }^{214} \mathrm{~Pb}$ de courte période $(26,8$ minutes) disparaît 3 heures environ après la séparation du radium. Donc, en absence du $214 \mathrm{~Pb}$, la raie $53,2 \mathrm{keV}$ est due uniquement à l.234 $\mathrm{U}$, ce qui permet de mesurer directement les rapports ${ }^{230} \mathrm{Th} /{ }^{234} \mathrm{U}$ et ${ }^{234} \mathrm{U} /{ }^{238} \mathrm{U}$ sans aucune correction d'interférences avec la raie 53,3 keV émise par le ${ }^{214} \mathrm{~Pb}$ et 10 fois plus intense que la raie émise par ${ }^{1234} \mathrm{U}$.

- Tous les rapports d'équilibre dans un échantillon sont normalisés aux mêmes rapports dans l'échantillon étalon. Les deux échantillons sont réduits, après attaque et élimination du radium, à des matrices identiques d'hydroxydes de fer ou d'aluminium contenant l'uranium et le thorium; cependant des corrections dues à l'autoabsorption dans les deux échantillons ne sont plus nécessaires.

Les 2 échantillons, ayant des matrices et des géométries pareilles, sont comptés dans les mêmes conditions; ce qui simplifie, dans le calcul des rapports d'équilibre, les facteurs de correction dus à la géométrie de comptage.

La spectrométrie gamma à basse énergie destructive permet donc de dater à partir du même spectre, par 2 méthodes de déséquilibre radioactif $\left({ }^{230} \mathrm{Th} /{ }^{234} \mathrm{U}\right.$ et $\left.{ }^{231} \mathrm{~Pa} /{ }^{235} \mathrm{U}\right)$. Cette technique peut être utilisée surtout pour

\section{A. Choukri et al.}


analyser les échantillons géologiques riches en uranium et qui posent un problème de pollution radioactive en spectrométrie alpha dans les laboratoires qui traitent les échantillons de faibles activités. Elle pourra être également étendue aux échantillons de faible activité tels que les coraux et les coquilles, en attaquant plus de matière sans changer la matrice d'hydroxydes de fer ou d'Aluminium.

\section{Références}

[1] - Y. Yokayama, H. V. Nguyen "Direct and non destructive dating of marine sediments, nianganese noduls, and corals by high resolution gamma-ray spectrometruy" Isotope Marine Chemistry, (E.D. Goldberg, Y. Horibe and K. Saruhashi, Eds.), Uchida Rokakuho Pub. Co., Lts., Japan (1980) 259-289.

[2] - Y. Nir-El "Dating the age of a nuclear event by gamma spectrometry" Applied Radiation and Isotopes, 60 (2004) 197-201.

[3] - J.-L. Reyss, S. Schmidt, F. Legeleux, P. Bonté Large, low background well-type detectors for measurements of environmental radioactivity, Nuclear Instruments and Methods in Physics Research Section A: Accelerators, Spectrometers, Detectors and Associated Equipment, 357 (1995) 391-397.

[4] - J.-L. Reyss, S. Schmidt, D. Latrouite, S. Floris "Age determination of crustacean carapaces using ${ }^{228} \mathrm{Th} /{ }^{228} \mathrm{Ra}$ measurements by ultra low level gamma spectroscopy" Applied Radiation and Isotopes, Volume 47, Issues 9-10 (1996) 1049-1053.

[5] - F. De Corte, H. Umans, D. Vandenberghe, A. De Wispelaere, P. Van den haute "Direct gamma-spectrometric measurement of the ${ }^{226} \mathrm{Ra} 186.2 \mathrm{keV}$ line for detecting ${ }^{238} \mathrm{U} /{ }^{226} \mathrm{Ra}$ disequilibrium in determining the environmental dose rate for the luminescence dating of sediments" Applied Radiation and Isotopes, 63 (2005) 589-598

[6] - S. Schmidt, J.-L. Reyss "Radium as internal tracer of Mediterranean Outflow Water" Journ. Geophys. Res., 101 (1996) 3589-3596. 
[7] - O.-K. Hakam "Etude de la répartition des activités des radioisotopes de l'uranium et du radium dans les eaux naturelles et du radon dans l'air des habitations au Maroc" Thèse d'état, Univ. Med $\mathrm{V}, \mathrm{N}^{\circ} 1893$ (2000) 212p.

[8] - O.-K Hakam., A. Choukri, J.-L. Reyss' M. Lferde " Comparaison des activités des radio-isotopes de l'uranium et du radium dans quelques échantillons d'eau de puits et de sources thermales au Maroc." Revue des Sciences de l'Eau, 13 (2000) 187-194

[9] - O.-K. Hakam, A. Choukri, J.L. Reyss, M. Lferde "Activities and activity ratios of $U$ and Ra radio-isotopes in drinking wells, springs and tap water samples in Morocco" Journal of Radiochemica Acta, 88 (2000) 55-60.

[10] - O.-K. Hakam, A. Choukri, J.-L. Reyss, M. Lferde "Determination and comparison of uranium and radium isotopes activities and activity ratios in samples from some natural water sources in Morocco" Journal of Environmental Radioactivity 5 (2001) 175-189.

[11] - A. Choukri "Application des méthodes de datation par les séries d'uranium à l'identification des hauts niveaux marins sur la côte égyptienne de la mer rouge en moyen de coraux, radioles d'oursins et coquilles, et sur la côte atlantique du Haut Atlas au Maroc, au moyen de coquilles. Thèse D'état, Univ Med V, Rabat, $\mathrm{N}^{\circ} 1184$ (1994) 192p.

[12] - M. Berrada, A. Choukri, T. El Khoukhi "Non destructive and destructive dating by low energy gamma ray spectrometry" Appl. Radiat. Isot. 46 (1995) 651-652. 